\title{
Tratamiento endovascular del síndrome de vena cava superior
}

\author{
Michel Bergoeing $\mathbf{R}^{1}$, Renato Mertens $M^{1}$, Francisco Valdés $E^{1}$, \\ Albrecht Krämer Sch ${ }^{1}$, Manuel Alvarez Z2 ${ }^{2}$, Pablo Bertin $C^{2}$, \\ Rodrigo Sagüés $C^{1}$, Eric O rellana $U^{2}$, Héctor $G$ alindo $A^{2}$, \\ Jeannette Vergara $\mathbf{G}^{1, a}$, Magaly Valdebenito $C^{1, a}$.

\section{Endovascular treatment of superior vena cava syndrome}

Background: Superior vena cava syndrome (SVCS) is caused by the obstruction of venous drainage from the upper portion of the body. Common clinical findings are headache and cervical, facial and upper limb edema. Occasionally, clouding of consciousness appears. Aim: to report our experience with endovascular treatment of SVCS. Material and methods: Retrospective review of all patients with SVCS subjected to endovascular treatment between 1999 and 2005. Results: Eight patients were treated, all of them with malignancies. Six had a benign obstruction due to the presence of a chemotherapy catheter located in the superior vena cava, one had obstruction secondary to radiation therapy and one a tumor compression of the superior vena cava. Two patients underwent thrombolytic therapy. Angioplasty and stenting was performed in all patients. The chemotherapy catheter was removed to all patients and installed again in one. One patient had a hemothorax secondary to a simultaneous needle lung biopsy under video thoracoscopy. No patient died in relation to the procedure. Congestive signs and symptoms subsided in all patients within 24 hours after the procedure. During follow up, only one patient had symptoms related to vena cava obstruction and three died due to their malignant tumor. Conclusions: Endovascular treatment of SVCS has a low rate of complications and provides immediate and mid-term symptom relief (Rev Méd Chile 2006; 134: 827-32).

(Key w ords: Angioplasty; Stents; Superior vena cava syndrome)

Recibido el 4 de julio, 2005. Aceptado el 10 de enero, 2006.

${ }^{1}$ Cirugía Vascular y Endovascular, Departamento de Enfermedades Cardiovasculares y ${ }^{2}$ Departamento de Hematología-Oncología, Facultad de Medicina, Pontificia Universidad Católica de Chile. Santiago de Chile.

aEnfermera Universitaria.

$\mathrm{E}^{1}$ síndrome de vena cava superior (SVCS) consiste en edema y congestión venosa del hemicuerpo superior, y es causada por la obstruc-

Correspondencia a: Dr. Renato Mertens M. Cirugía Vascular y Endovascular, Departamento de Enfermedades Cardiovasculares. Hospital Clínico Pontificia Universidad Católica de Chile. Marcoleta 367, $6^{\circ}$ piso. E-mail: rmertens@med.puc.cl. ción del retorno venoso a través de este vaso. La gran mayoría se debe a compresión tumoral, siendo la causa más frecuente una neoplasia pulmonar $^{1,2}$. Las causas benignas, si bien son menos frecuentes, actualmente, con el aumento del uso de catéteres permanentes o semi permanentes y marcapasos, han aumentado su frecuencia relativa, progresivamente ${ }^{3}$. La reparación tradicional implica una cirugía mayor con la construcción de 
un puente veno-venoso $0^{4,5}$, alternativa viable para un número reducido de pacientes con compromiso neoplásico de la vena cava superior $(\mathrm{VCS})^{6-8}$. En los últimos años se ha desarrollado la cirugía endovascular, como una alternativa mínimamente invasiva, efectiva y de baja morbilidad, en especial en pacientes con etiología neoplásica ${ }^{9-18}$. El presente trabajo muestra la experiencia con una serie de 8 pacientes intervenidos por SVCS, tratados en forma percutánea.

\section{MATERIAL Y MÉTODO}

Se revisó, en forma retrospectiva, la ficha de todos los pacientes portadores de alguna neoplasia e intervenidos por vía endovascular por SVCS en nuestro servicio, entre los años 1999 y 2005. Todos ellos referidos por el grupo de oncología, al fracasar las medidas de manejo médico habituales. Se consignaron las características demográficas, clínicas, etiológicas, complicaciones observadas y mortalidad hospitalaria o a los 30 días. Se registró el tratamiento médico utilizado, el tiempo de resolución de los síntomas y se realizó seguimiento completo a todos los pacientes a través de entrevista personal, control telefónico o a través del Servicio de Registro Civil e Identificación, en caso de fallecimiento. Se consideró como éxito técnico el lograr el cruce de la lesión obstructiva, la apertura del lumen a un mínimo de $10 \mathrm{~mm}$ de diámetro, ausencia de reflujo proximal durante la cavografía de control y flujo preferencial a través de la vena cava y no por el lecho colateral. El resultado clínico de la reparación fue evaluado en base a la resolución completa de los signos y síntomas obstructivos que motivaron la intervención.

\section{RESULTADOs}

Se trataron 8 pacientes, $7(87,5 \%)$ de sexo femenino. La edad promedio fue $52,4 \pm 12$ años (32-68). Las características demográficas de los pacientes y etiología del SVCS se detallan en la Tabla 1. En los enfermos con etiología secundaria a la presencia de un catéter reservorio, el tiempo de permanencia del catéter varió entre 1 año 3 meses y 5 años. Los síntomas asociados se muestran en la Tabla 2. En todos, el diagnóstico clínico se confirmó mediante tomografía axial computada.

Todos los pacientes fueron intervenidos en un pabellón quirúrgico y se utilizó un angiógrafo con sustracción digital (Siremobil, Siemens ${ }^{\circledR}$; OEC9800, General Electric ${ }^{\circledR}$ ). A todos se les practicó cavografía (Figura 1). Debido a la presencia de trombo en la vena cava superior, en dos pacientes se realizó trombolisis local (urokinasa o activador del plasminógeno tisular) durante $24 \mathrm{~h}$, completando el procedimiento posteriormente. La angioplastia fue realizada bajo anticoagulación sistémica con heparina. Se realizó el cruce de la lesión con una guía hidrofílica. En cuatro pacientes practicamos angioplastia primaria con stent balón expandible. Los otros cuatro fueron predilatados con balón, seguido del despliegue de un stent autoexpansible, seguido de postdilatación (Figura 2). El acceso fue vía vena yugular en $5(62,5 \%)$, vena femoral en dos (25\%) y vena cefálica en uno (12,5\%). A todos los

Tabla 1. C aracterísticas y diagnóstico de los 8 pacientes intervenidos

\begin{tabular}{|ccccc|}
\hline $\mathrm{N}^{\circ}$ paciente & Sexo & Edad & Etiología & Neoplasia \\
\hline 1 & Femenino & 49 & Catéter reservorio & Mieloma múltiple \\
2 & Masculino & 68 & Catéter reservorio & Gástrico \\
3 & Femenino & 53 & Tumoral & Pulmonar \\
4 & Femenino & 64 & Catéter reservorio & Mama \\
5 & Femenino & 61 & Catéter reservorio & Mama \\
6 & Femenino & 32 & Catéter reservorio & Mama \\
7 & Femenino & 51 & Catéter reservorio & Mama \\
8 & Femenino & 41 & Act́nica & Timoma \\
\hline
\end{tabular}


Tabla 2. Signos y síntomas asociados a la obstrucción de la vena cava superior

\begin{tabular}{|lcc|}
\hline Signo o síntoma & Número & $\%$ \\
\hline Edema facial & 8 & 100 \\
Edema extremidades superiores & 5 & 62,5 \\
Cianosis & 2 & 25 \\
Congestión nasal & 2 & 25 \\
Circulación colateral superficial & 2 & 25 \\
Edema palpebral & 1 & 12,5 \\
Cefalea & 1 & 12,5 \\
Dolor ocular & 1 & 12,5 \\
Tos irritativa & 1 & 12,5 \\
\hline
\end{tabular}

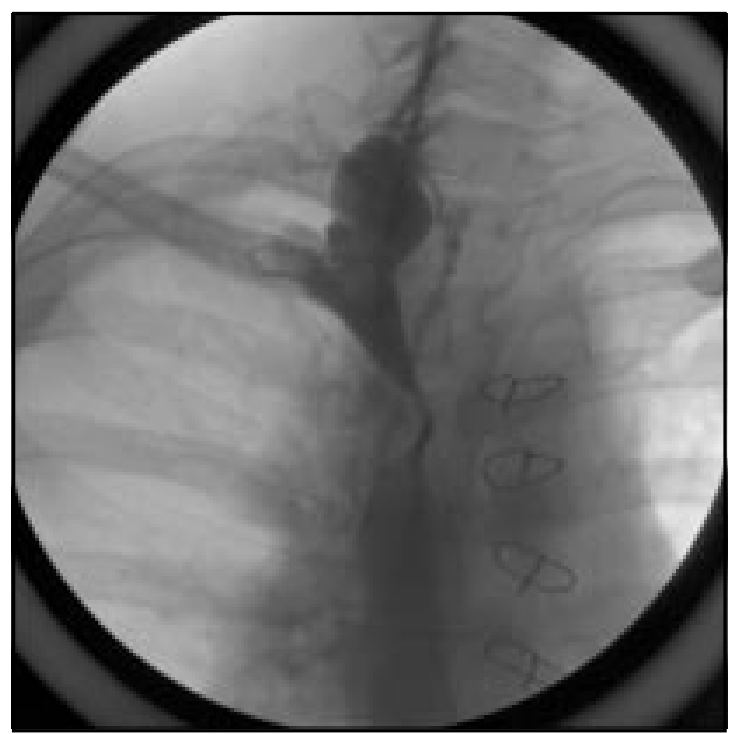

Figura 1. Cavografía. Notar colateral a través del sistema hemiácigos.

portadores de un catéter reservorio se les retiró éste durante la angioplastia, siendo necesario implantar uno nuevo en un enfermo, para continuar su quimioterapia. A un paciente se le practicó una biopsia pulmonar bajo video-toracoscopia por sospecha de metástasis, en forma concomitante con la angioplastia.

La evolución postoperatoria fue sin incidentes en 7 pacientes. El paciente sometido a biopsia

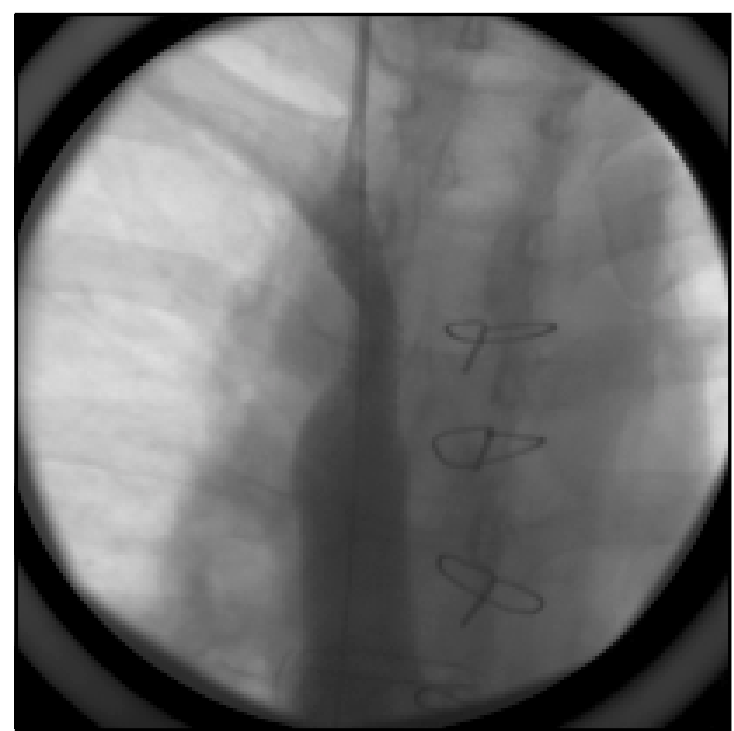

Figura 2. Post despliegue stent. Notar la desaparición de la circulación colateral.

pulmonar presentó un hemotórax, que requirió drenaje mediante una nueva video-toracoscopia, siendo dado de alta al sexto día en buenas condiciones. No hubo otra morbilidad ni mortalidad asociada al procedimiento. Todos los pacientes presentaron regresión de sus síntomas a las primeras $24 \mathrm{~h}$ post procedimiento (Figuras $3 \mathrm{~A}$ y $3 B$ ). La estadía promedio fue $2,4 \pm 1,6$ días (rango 1-6). Cinco pacientes recibieron clopidogrel y 3 


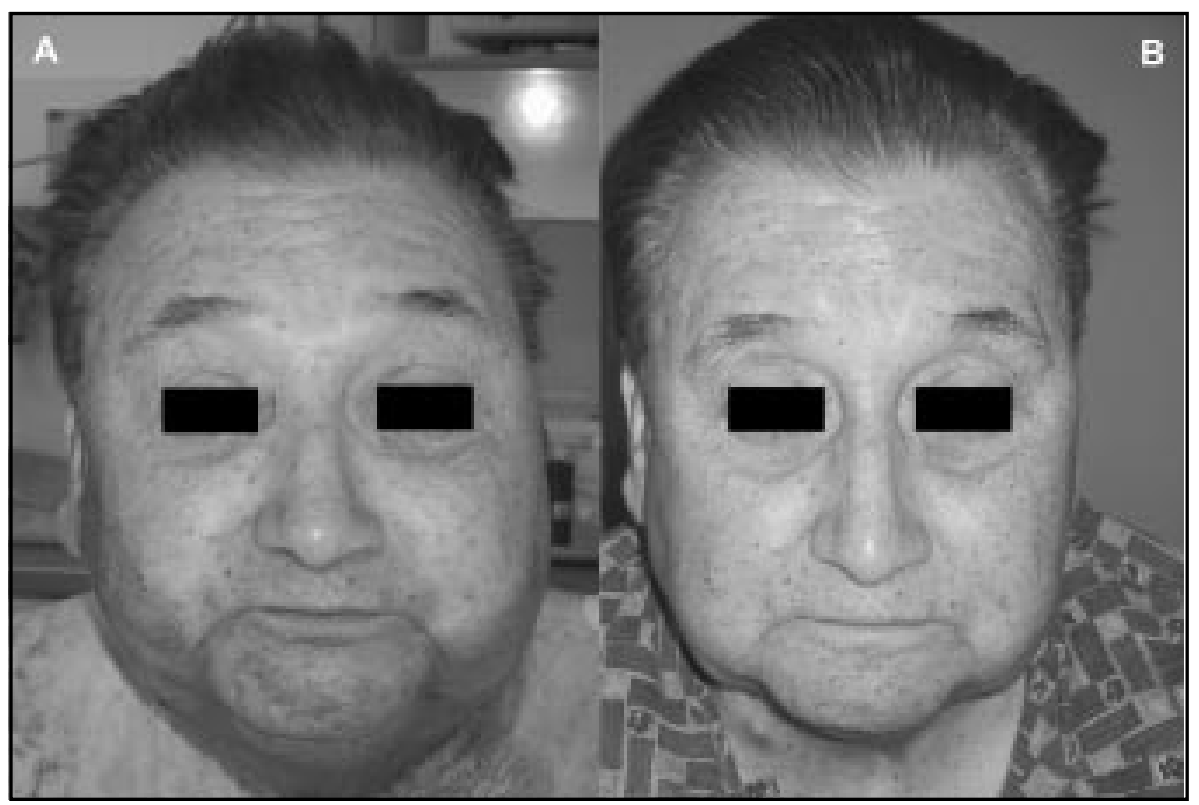

Figura 3. A: Preangioplastia. Notar edema facial y palpebral. B: Una semana postangioplastia.

anticoagulación oral con acenocumarol al momento del alta, por al menos 3 meses. El seguimiento promedio fue $5,7 \pm 3,8$ meses (rango 1,1-10,4). Tres pacientes fallecieron durante el seguimiento, debido a su neoplasia de base, en uno de los cuales recidivó la sintomatología obstructiva a los 38 días post angioplastia. Debido a las malas condiciones generales del paciente, se manejó en forma paliativa, falleciendo 10 días después. El resto de los pacientes se encuentra en buenas condiciones y asintomáticos al momento del control.

\section{Discusión}

El SVCS es un cuadro altamente incapacitante y potencialmente letal, que en $80 \%$ de los casos se presenta en pacientes portadores de una neoplasia pulmonar o mediastínica ${ }^{1}$, y que empeora significativamente la ya deteriorada calidad de vida de estos pacientes. El 20\% restante se debe a causas benignas, las que han ido en aumento debido al uso masivo de accesos venosos centrales ${ }^{3,13}$. En un estudio anatomopatológico de pacientes portadores de accesos venosos centrales ${ }^{19}$, a los dos días de instalado el catéter ya son visibles cambios inflamatorios de la íntima y media con formación de trombos; y en pacientes con más de 90 días, se observa engrosamiento de la pared venosa con alteración del plano elástico y muscular, además de adherencias entre el catéter y la pared venosa formados por trombo, colágeno y pseudoíntima. En los pacientes portadores de alguna neoplasia hay que considerar, además, el estado protrombótico que éstas causan en forma secundaria. Estos datos son concordantes con nuestra experiencia: en seis pacientes (75\%), la etiología del SVCS fue la presencia de un acceso venoso central para quimioterapia de larga duración.

El manejo tradicional de este cuadro incluye terapia médica en base a cambios posicionales, diuréticos, anticoagulación y corticoides; radioterapia, quimioterapia o ambos $1,2,12,13,17$. El resultado de estos tratamientos no siempre es efectivo y la respuesta terapéutica no es evidente antes de la segunda o tercera semana. Otra alternativa es la cirugía de reconstrucción venosa mediante puente entre las venas yugular derecha o innominada izquierda y la aurícula derecha, la que requiere de una toracotomía o esternotomía, con buenos resultados a largo plazo en etiología benigna ${ }^{4,5}$. Sin 
embargo, en casos de etiología maligna, la morbilidad y la mortalidad de la cirugía varían entre 23$39 \%$ y $12-14 \%$, respectivamente, lo que parece desproporcionado en un paciente cuya mediana de sobrevida es de 9 meses $^{6-8}$. En todo caso, esta opción permanece disponible luego del tratamiento endovascular y un eventual fracaso de éste.

Desde la publicación de Charnsangavej20 en 1986, sobre su experiencia con el uso de stents metálicos para el manejo de la obstrucción de la vena cava en perros, numerosas publicaciones han dado cuenta de excelentes resultados clínicos en seres humanos ${ }^{9-18}$, incluyendo nuestro primer caso, reportado en $2001^{15}$. El procedimiento se asocia a una muy baja tasa de morbimortalidad, se realiza bajo anestesia local y no implica una incomodidad mayor para el paciente. La morbilidad y la mortalidad descritas varían entre $0 \%-23 \%$ y $0 \%-6,7 \%$, respectivamente $9-18$. Dentro de las complicaciones se cuentan migración del stent ${ }^{11,14,16}$, edema pulmonar por aumento del retorno venoso $0^{9,14,21}$, embolia pulmonar ${ }^{13,14}$, trombosis aguda del stent ${ }^{11,12,16}$ y hemorragia ${ }^{12-}$ 14 , entre otras. En todas las series reportadas, el éxito técnico fluctúa entre $95 \%$ y $100 \%$, el éxito clínico 90\%-100\%, la permeabilidad primaria 77\%$92 \%$ y la permeabilidad secundaria $85 \%-100 \%{ }^{9-18}$, con un seguimiento que varía entre 2,9 y 17 meses.

\section{REFERENCIAS}

1. Shimm D, Logue G, Rigsby L Evaluating the superior vena cava syndrome. JAMA 1981; 245: 951-3.

2. ABNER A. Approach to the patient who presents with superior vena cava obstruction. Chest 1993; 103: 394S-397S.

3. Sharafuddin M, Sun S, Hobaliah J. Endovascular management of venous thrombotic diseases of the upper torso and extremities. J Vasc Interv Radiol 2002; 13: 975-90.

4. Gloviczki P, Pairolero P, Cherry K, HaLet J. Reconstruction of the vena cava and of its primary tributaries: a preliminary report. J Vasc Surg 1990; 11: 378-81.
En nuestra serie, todos los pacientes, excepto uno, fueron intervenidos bajo anestesia local, con un éxito técnico y clínico inmediato de $100 \%$. No hubo mortalidad perioperatoria y sólo un paciente presentó morbilidad derivada de un procedimiento asociado. Durante el seguimiento, sólo un enfermo $(12,5 \%)$ evidenció recidiva de los síntomas poco antes de fallecer. No se practicó necropsia. La etiología del SVCS en este paciente era compresión tumoral de la VCS. El principal factor pronóstico de permeabilidad de la reparación de la VCS es la presencia de invasión tumoral ${ }^{11}$. Para estos casos, se ha reportado el uso de stents cubiertos, con buenos o mejores resultados ${ }^{22,23}$.

Habitualmente, el seguimiento de estos pacientes es corto, ya que la mayoría sucumbe a su enfermedad de base. Nuestro caso no es distinto. Casi la mitad de los pacientes falleció por su neoplasia. A pesar de esto, la angioplastia les confiere una mejoría en su calidad de vida con una muy baja morbimortalidad. En casos de recidiva, ésta puede ser manejada exitosamente por vía endovascular.

La cirugía endovascular del SVCS es una alternativa de bajo riesgo y excelentes resultados en el alivio sintomático de estos pacientes. Actualmente debiera ser considerada como primera línea de tratamiento para el manejo de esta patología.

5. Gloviczki P, Pairolero P, Toomey B, Bower T, Rooke T, Stanson A ET AL. Reconstruction of lange veins for nonmalignant venous occlusive disease. J Vasc Surg 1992; 16: 750-61.

6. Spaggiari L, Thomas P, Magdeleinat P, Kondo $\mathrm{H}$, Rolet G, Regnard J et al. Superior vena cava resection with prosthetic replacement for nonsmall cell lung cancer: long-term results of a multicentric study. Eur J Cardiothorac Surg 2002; 21: $1080-6$.

7. Spaggiari L, Magdeieinat $\mathrm{P}, \mathrm{Kondo} \mathrm{H}$, Thomas $\mathrm{P}$, Leon M, Rowet G et al. Results of superior vena cava resection for lung cancer. Analysis of prognostic factors. Lung Cancer 2004; 44: 339-46.

8. Shargall $Y$, De Perrot M, Keshavjee S, Darling G, GinsBerg R, Johnston M et al. 15 years single 
center experience with surgical resection of the superior vena cava for non-small cell lung cancer. Lung Cancer 2004; 45: 357-63.

9. Kishi K, Sonomura T, Mitsuzane K, Nishida N, Yang R, Sato M et aL. Self expandable metallic stent therapy for superior vena cava syndrome: clinical observations. Radiology 1993; 189: 531-5.

10. Dodds A, Harrison J, O’Laughin M, Wilson J, KISSLO K, BASHORE T. Relief of superior vena cava syndrome due to fibrosing mediastinitis using the palmaz stent. Chest 1994; 106: 315-8.

11. Furui S, Sawada S, Kuramoto K, Inoue Y, Irie T, Makita $\mathrm{K}$ ET AL. Gianturco stent placement in malignant caval obstruction: analysis of factors for predicting outcome. Radiology 1995; 195: 147-52.

12. Hennequin L, Fade O, Fays J, Bic JF, JaAfar S, Bertal A ET AL. Superior vena cava stent placement: results with the wallstent endoprosthesis. Radiology 1995; 196: 353-61.

13. Nicholson A, EtTles D, Arnold A, Greenstone M, DyET J. Treatment of malignant superior vena cava obstruction: metal stents or radiation therapy. J Vasc Interven Radiol 1997; 8: 781-8.

14. Kee S, Kinoshita L, Razavi M, Nyman U, Semba C, DAKE M. Superior vena cava syndrome: treatment with catheter-directed thrombolysis and endovascular stent placement. Radiology 1998; 206: 187-93.

15. Boza C, Mertens R, Valdés F, KRÄmer A, Marine L, Vergara J. Terapia endovascular en el síndrome de vena cava superior: caso clínico. Rev Méd Chile 2001; 129: 421-6.
16. Lanciego C, Chacón JL, Julúán A, Andrade J, López L, Martínez B ET AL. Stenting as first option for endovascular treatment of malignant superior vena cava síndrome. AJR 2001; 177: 585-93.

17. Kvale P, Simoff M, Prakash U. Palliative Care. Chest 2003; 123: 284S-311S.

18. KIM YI, Kim KS, Ko YC, PARK CM, LIM SC, KIM YC ET AL. Endovascular stenting as a first choice for the palliation of superior vena cava syndrome. J Korean Med Sci 2004; 19: 519-22.

19. Forauer A, Theoharis $C$. Histologic changes in the human vein wall adjacent to indwelling central venous catheters. J Vasc Interven Radiol 2003; 14: 1163-8.

20. Charnsangavej C, Carrasco H, Wallace S, Wright K, OgaWa K, Ruchu W et al. Stenosis of the vena cava: preliminary assessment of treatment with expandable metallic stents. Radiology 1996; 161: 295-8.

21. Yamagami T, NaKamura $T$, Kato T, Iida S, Nishimura T. Hemodynamic changes after self-expandable metallic stent therapy for vena cava syndrome. AJR 2002; 178: 635-9.

22. Chin D, Petersen B, Timmermans H, Rosch J. Stentgraft in the management of superior vena cava syndrome. Cardiovasc Intervent Radiol 1996; 19: 302-4.

23. GiL K, Ettles D, Nicholson A. Recurrent superior vena caval obstruction due to invasion by malignant thymoma: treatment using a stent-graft. $\mathrm{Br} \mathrm{J}$ Radiol 2000; 73: 1015-7. 